\title{
sciendo
}

Transport and Telecommunication, 2019, volume 20, no. 1, 1-11

Transport and Telecommunication Institute, Lomonosova 1, Riga, LV-1019, Latvia DOI 10.2478/ttj-2019-0001

\section{ANALYSIS OF THE HYBRID GLOBAL PATH PLANNING ALGORITHM FOR DIFFERENT ENVIRONMENTS}

\author{
Paulius Skačkauskas ${ }^{1}$, Edgar Sokolovskij $^{2}$ \\ ${ }^{1,2}$ Department of Automobile Engineering, \\ Vilnius Gediminas Technical University \\ Basanavičiaus g. 28, LT-03224, Vilnius, Lithuania \\ I paulius.skackauskas@vgtu.lt, ${ }^{2}$ edgar.sokolovskij@vgtu.lt
}

To achieve the overall goal of realising an efficient and advantageous participation of autonomous ground vehicles in the transport system as fast as possible, a lot of work is being done in different and specific research fields. One of the most important research fields, which has a large impact on safe autonomous ground vehicle realisation, is the development of path planning algorithms. Therefore, this work describes in detail the development and application of a hybrid path planning algorithm. The described algorithm is based on classical and heuristic path planning approaches and can be applied in unstructured and structured environments. The efficiency of the algorithm was investigated by applying the algorithm and executing theoretical and experimental tests. The theoretical and experimental tests were executed while optimising different complexity paths. Results analysis demonstrated that the described algorithm can generate a smooth, dynamically feasible and collision-free path.

Keywords: autonomous vehicle, path planning, hybrid algorithm, optimisation, structured environment, unstructured environment

\section{Introduction and Literature Review}

As modern active safety systems in the last decade increased traffic safety noticeably, the common interest of the general public in the field of autonomous ground vehicles (AGVs) keeps growing. However, to this day AGVs are still not developed enough to reliably increase traffic safety, while autonomously performing in different environments. Zhang et al. (2018) noted that as a key module to autonomous driving system, path and trajectory planning algorithms play an important role in guaranteeing ride safety and comfort by generating a smooth, dynamically feasible, collision-free trajectory, while taking into account various specific constraints. Thus, seeking to further increase traffic safety, research progress in the study field of AGVs path and trajectory planning has to be more rapid and due to this reason various path planning algorithms are being developed in research works. Based on the algorithm approach, Mac et al. (2017) singled out two types of path planning algorithms: classical algorithms and heuristic based algorithms. The classical path planning algorithms refer to geometric algorithms and graph search. The heuristic algorithms include neural network, fuzzy logic and other optimisation based techniques (Mac et al., 2017). M. Samadi and M.F. Othman (2013) also remarked that the path planning algorithms can be divided into another two categories, which are local and global algorithms. In the case of local path planning problems, the environment model is defined as dynamic, while, in the case of global path planning, the environment should be defined as static. The literature review in this work further focuses on global path planning algorithms and their problems.

Due to the reason that each of the approaches has its own limitations, one individual algorithm cannot ensure an optimal, i.e. dynamically feasible and smooth, collision-free, etc. path (Mac et al., 2017). Thus, researchers have been seeking to develop more sophisticated and hybrid algorithms. For example, an innovative algorithm, based on A-star algorithm, called a relaxed A-star algorithm, which has linear complexity with relaxed constraints is presented in (Chaari et al., 2017). The authors state that due to some computation elements of the classic A-star algorithm being removed, the relaxed A-star algorithm provides near optimal solutions with reduced execution time as compared to the classic A-star algorithm. In various research works different improvements of the A-star algorithm, like the dynamic Astar or the repairing A-star algorithm, can be found, however, Gonzalez et al. (2015) pointed out that the main disadvantage of the A-star based algorithms - the resulting path is not continuous. An extension of another classical path planning algorithm - rapidly-exploring random trees (RRTs), is developed in (Paniagua et al., 2018). The extended algorithm - quad-RRT, computes four trees instead of two, this way 
speeding up the classic RRT, however it requires more hardware and computational resources than the other types of RRT based algorithms. A review on different RRT approaches is given in (Noreen et al., 2016). According to (Noreen et al., 2016), though the RRTs gained immense popularity due to its support for high dimensional complex problems, slow convergence and high memory requirements are still open research issues for the RRT algorithms. To solve the problem of global path planning, researchers in (Araujo de Lemos et al., 2015) used a splines based algorithm. Novelty of the splines based algorithm, presented in (Araujo de Lemos et al., 2015) - it works in the arc length space instead of the time space, which allows the generation of the candidate paths without the profile velocity. A heuristic and optimisation, i.e. a genetic algorithm based global path planner is presented and investigated in (Samadi and Othman, 2013). In the study it was stated that the special genetic algorithm crossover and selection functions proposed by the authors, performs better in terms of path length and calculation time, compared with the conventional genetic algorithm. Lamini et al. (2018) also improved the crossover operator of the genetic algorithm, for solving path planning problems in static environments. The proposed improved crossover operator of the genetic algorithm in (Lamini et al., 2018) avoids premature convergence and an optimal path is found in less iterations compared to the other methods. Another heuristic nature-inspired optimisation algorithm for global path planning - particle swarm optimisation, is used in (Mac et al., 2017). The authors proposed a particle swarm optimisation with an accelerated update methodology based on the Pareto dominance principle for multi-objective path planning problem. An advantage of the proposed particle swarm optimisation based algorithm - it is significantly fast in optimisation. A global path planning algorithm based on improved ant colony optimisation is presented in (Lee et al., 2011). In their research Lee et al. (2011) indicated that ants carry out the exploration task relatively well around the starting point of the global path, however, they have a strong tendency not to explore and most of them follow the path found in the beginning of the search. To take advantage of the strengths of some approaches while reducing the effects of their drawbacks, some researchers have combined two or more algorithms in their investigations to provide efficient hybrid algorithms for path planning and control of AGVs (Ayawli et al., 2018). Elhoseny et al. (2018) introduced a Bezier curve based global path planning, while using a modified genetic algorithm. In (Elhoseny et al., 2018) the standard genetic algorithm was modified by increasing its exploration capability. Respectively, the goal of the modified genetic algorithm was to search for the most suitable points as the control points of the Bezier curve. To generate an optimal path, in (Zhou and Gon, 2013) a novel RRT and particle swarm optimisation based algorithm is proposed. The analysis of the algorithm demonstrated that the algorithm performs reliably and convergences efficiently. In an overview work by Ayawli et al. (2018) it is accented that even though there are challenges while using the hybrid algorithms, due to the reason that the hybrid algorithms seem to possess the strengths of the algorithms integrated while reducing their drawbacks, it can be considered to obtain better techniques for path planning of AGVs. Taking into consideration the assumption that the development and application of the hybrid path planning algorithms is a key factor to achieve safe and efficient path planning algorithms for AGVs and respectively, to increase traffic safety, the aim of this work is to further investigate and evaluate practical application possibilities of the hybrid path planning algorithm, recently developed by the authors of this work.

\section{Hybrid Global Path Planning Algorithm}

The proposed hybrid global path planning algorithm is combined from both, classical - Dubins path, and heuristic - genetic algorithm approaches. Respectively, the generation of the path consists of two stages. Firstly, a primary coarse path, made of straight-line segments, is converted into a Dubins path by applying the Dubins path approach. Secondly, the Dubins path is optimised by applying the genetic algorithm. The application of the proposed path planning algorithm is explained in order further:

- Unlike in a large number of algorithms proposed by various researchers, in which the primary coarse path is generated based on the first and the final waypoints of the path, in this work it is assumed that the primary coarse path is predefined by a human user and consists of straight-line segments and sharp turns. Thus, a first set of waypoints $\left(w_{i}\right)$, that defines the straight lines and turning points in structured and unstructured environments, is implemented into the algorithm.

- In the case when the proposed algorithm is going to be applied to the path planning in the structured environments, a second set of waypoints $\left(d_{i}\right)$, which defines road network or other static obstacles geometry, also must be implemented. Commonly, in various research works, to define the environment of $\mathrm{AGV}$, an additional environmental model is applied. However, seeking to ensure simplicity and efficiency of the path planning process, in this work, the first 
and second sets of waypoints are implemented into the proposed algorithm while using a satellite map of the required structured environment. It is assumed that the environment is static and well known.

- To perform the path re-planning and generate a smooth path without sharp turns, the Dubins path approach is selected. This classic path planning approach is selected because of its efficiency and small computational resources requirement. While applying this approach, the primary coarse path is converted into the Dubins path by changing the waypoints of the first set into a filleted circular arc with minimum turning radius $\left(r_{\min }\right)$, of AGV. Thus, the steady state cornering condition is accepted. The Dubins path approach, which is further applied in this work, was initially described in detail for the use of unmanned aerial vehicles obstacle avoidance in (Kikutis et al., 2017) and should be applied as follows.

Unit vectors $\left(q_{i}\right)$, which indicate moving directions of AGV in every straight-line segment of the primary coarse path, are defined:

$q_{i}=\frac{w_{i+1}-w_{i}}{\left\|w_{i+1}-w_{i}\right\|}, q_{i+1}=\frac{w_{i+2}-w_{i+1}}{\left\|w_{i+2}-w_{i+1}\right\|}$.

Angle $\left(\alpha_{i}\right)$ between the adjacent straight-line segments:

$\alpha_{i}=\cos ^{-1}\left(-q_{i}^{T} \cdot q_{i+1}\right)$.

The transition distances $\left(k_{i}\right)$ from the waypoints of the first set to the transition points $\left(H_{i}\right)$, where filleted circular arcs with minimum turning radius of $\mathrm{AGV}$ must be inserted, are expressed:

$k_{i}=-\left(\frac{r_{\min }}{\tan \frac{\alpha_{i}}{2}}\right) q_{i+1}$.

Then, the coordinates of the transition points $\left(H_{i}\right)$ are identified:

$H_{i}\left(w_{i+1}-k_{i}, q_{i}\right), H_{i+1}\left(w_{i+1}+k_{i}, q_{i+1}\right)$.

The centre point coordinates $\left(C_{i}\right)$ of filleted circular arcs with minimum turning radius of AGV, also called Dubins circles, are determined:

$$
C_{i}=w_{i+1}-\left(\frac{r_{\min }}{\sin \frac{\alpha_{i}}{2}}\right) \frac{q_{i}-q_{i+1}}{\left\|q_{i}-q_{i+1}\right\|} .
$$

It is important to note that the given Dubins path approach should be applied in similar sequence to every waypoint from the first set, except for the first and the final waypoints, which mark the beginning and the end of the path.

- In order to provide a dynamically feasible path, the generated Dubins path must be optimised. This statement can be explained by the fact that the Dubins path approach is based on the assumption that an AGV always has a minimum tuning radius. For proper understanding of the proposed algorithm, it must be pointed out that in this work the purpose of optimising the Dubins path is not to generate a large number of paths that eventually will be discarded, but to solve the optimisation based path planning problem and directly find the optimal path. Due to its convenience and significant performance, the genetic algorithm as an optimisation technique, is selected. Seeking to apply the genetic algorithm, an objective function was developed: 


$$
\min f=\sum\left(x-x_{r}\right)^{2}+\sum\left(y-y_{r}\right)^{2}+\sum\left(\left(\frac{v_{i} \cdot \tan \left(\tan ^{-1}\left(\frac{L}{r_{i}}\right)\right) \cdot t_{i}}{\left.\left.\sqrt{1+\frac{l_{r}^{2} \cdot \tan \left(\tan ^{-1}\left(\frac{L}{r_{i}}\right)\right)}{L}}\right)+\theta_{i-1}-\theta_{r}\right)}\right)^{2}\right.
$$

where $x, y$ are the position coordinates of the centre of mass of the AGV and $x_{r}, y_{r}, \theta_{r}$ are the reference coordinates of the generated Dubins path; $v_{i}$ is the constant reference velocity of the AGV, at which it should finish the section of the optimised path; $L$ is the wheelbase of the AGV; $l_{r}$ is the distance from the centre of the mass to the rear axle of the AGV; $t_{i}$ is the reference moving time from one transition point to the next transition point; $\theta_{i-1}$ is the $\mathrm{AGV}$ orientation at the previous transition point.

- The optimisation of the Dubins paths while only using the developed objective function (Eq. 6) does not ensure that the generated path will be dynamically feasible. Therefore, in the proposed algorithm, the environment structure, the nonholonomic and other constraints of the AGV are considered by formulating inequality constraints for the solving of the optimisation problem.

The lower and upper bounds for the optimised turning radius $r_{i}$, which cannot be exceeded due to the technical AGV limits, are defined:

$$
-r_{\text {max }} \leq r_{i} \leq r_{\text {max }},
$$

where $r_{\text {max }}$ is the maximal possible turning radius, defined by the maximal steering angle of the front wheels of the AGV.

The developed inequality constraints for the unstructured environments:

$\sqrt{\frac{r_{i}^{2} \cdot q_{i}(x)}{\tan \left(\frac{\alpha_{i}}{2}\right)^{2}}+\frac{r_{i}^{2} \cdot q_{i}(y)}{\tan \left(\frac{\alpha_{i}}{2}\right)^{2}}}-\frac{q_{i-S}}{2} \leq 0$,

$\sqrt{\frac{r_{i}^{2} \cdot q_{i}(x)}{\tan \left(\frac{\alpha_{i}}{2}\right)^{2}}+\frac{r_{i}^{2} \cdot q_{i}(y)}{\tan \left(\frac{\alpha_{i}}{2}\right)^{2}}}-\left(\frac{q_{i-S}}{2}-\frac{\tan ^{-1}\left(\frac{L}{r_{i}}\right) \cdot v \cdot S_{R}}{\omega_{i}}\right) \leq 0$,

where $q_{i}(x, y)$ is the length of the corresponding unit vectors in the $x$ and $y$ directions; $q_{i-S}$ is the length of the shorter straight line segment, connected to the corresponding waypoint of the first set; $S_{R}$ is the steering ratio of the $\mathrm{AGV}$ steering system; $\omega_{i}$ is the angular velocity of the steering wheel turning in different sections of the path.

Both inequality constraints, i.e. Eq. (8.1) and Eq. (8.2) can be used under the same conditions during optimisation. The main difference between these constraints is that Eq. (8.2) also takes into the consideration the reference velocity of the $\mathrm{AGV}$ and the angular velocity of the steering wheel turning. Thus, the value of the optimised turning radius $r_{i}$ will be lesser when using Eq. (8.2). Based on Eq. (8.2), the inequality constraint for the structured environments is formulated:

$r_{i}-\left(I_{i}+\frac{1}{2} \cdot \frac{\tan \left(\frac{L}{r_{i}}\right) \cdot v_{i} \cdot S_{R}}{\omega_{i}}\right) \leq 0$,

where $I_{i}$ is the distance between the corresponding first and the second set waypoints; $r_{i-1}$ is the value of the optimised turning radius at the previous waypoint; $w_{d}$ is the distance between the waypoints of the first set (lengths of the corresponding straight-line segments). 
- The inequality constraint given in Eq. (9) only takes into account the road network or other static obstacles geometry and not its limitations. To solve this problem, in this work, a new inequality constraint, which evaluates limitations of the structured environments, is formulated and given. The new formulated inequality constraints must be applied together with Eq. (9) as a system of inequality constraints for the structured environments. Formulated new inequality constraint:

$\sqrt{\frac{r_{i}^{2} \cdot q_{i}(x)}{\sin \left(\frac{\alpha_{i}}{2}\right)^{2}}+\frac{r_{i}^{2} \cdot q_{i}(y)}{\sin \left(\frac{\alpha_{i}}{2}\right)^{2}}}-r_{i}+S D_{i}-T_{i} \leq 0$,

where $S D_{i}$ is the minimal safety distance; $T_{i}$ is the distance between the corresponding waypoint of first set to the structured environment limitation during the turning manoeuvre.

As can be seen from Eq. (6-10), the optimisation of every turning radius separately, the evaluation of the reference velocity of the $\mathrm{AGV}$ and other parameters, like the angular velocity of the steering wheel turning, the steering ratio of the $\mathrm{AGV}$, etc., ensure that the planned path will be not only smooth and dynamically feasible, but also makes it possible to use this algorithm for practical applications. In order to ensure effective operation of the introduced algorithm, the algorithm should be applied for the structured and unstructured environments in the exact same pattern, step by step as described.

\section{Application of the Algorithm}

The theoretical and experimental application of the algorithm was executed in two stages, while using an autonomous ground test vehicle with kinematic control strategy (Fig. 1). During the first stage, the algorithm was applied for the path planning in unstructured environments. The aim of the application of the algorithm in this stage was to investigate the performance of the algorithm while executing completely different manoeuvres. Thus, three different manoeuvres were executed: a simple turn, a turnaround and a more complex manoeuvre, which consists of four different turns. During the second stage, the algorithm was applied for the path planning in structured environments, while re-creating the manoeuvring in four different intersections. The aim of the application of the algorithm in the second stage was to analyse and assure that the performance of the newly formulated system of inequality constraints is proper and also suitable for practical application. Respectively, two intersections were selected while seeking to re-create turning to the left, and two intersections were selected while seeking to re-create turning to the right.

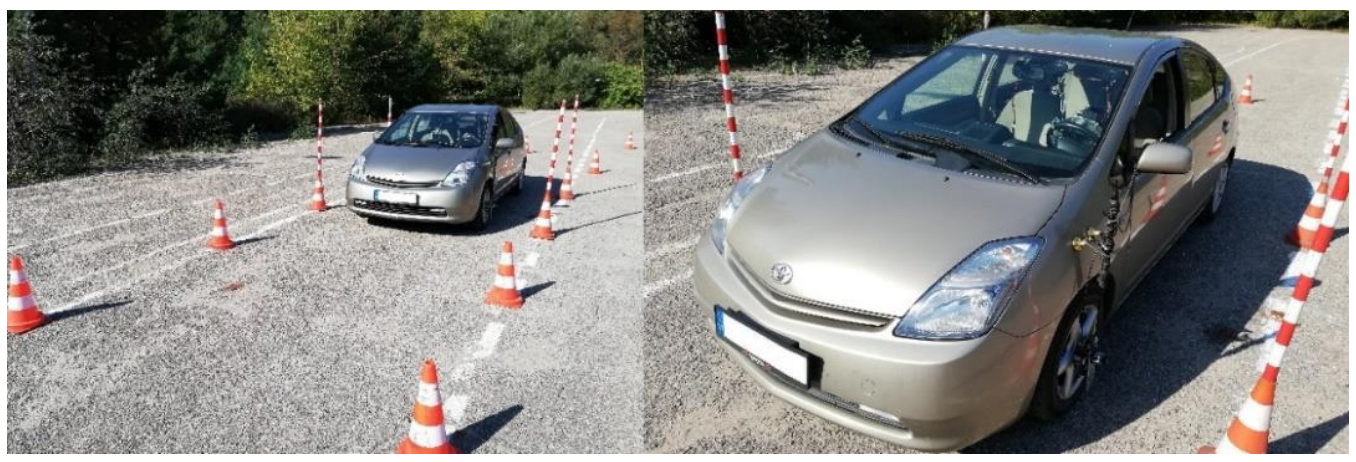

Figure 1. Autonomous ground test vehicle during experimental application of the proposed algorithm

In both stages the experimental application of the algorithm was executed in a publicly inaccessible driving lot. The experimental drives of every manoeuvre were repeated 4 times each. The wheelbase $L$ of the used AGV was 2.7 meters, the distance $l_{r}$ from the centre of the mass to the rear axle of the AGV - 1.4 meters. The predefined angular velocity value of the steering wheel turning in every manoeuvre $-2.8 \mathrm{rad} / \mathrm{s}$. The assumed reference velocity of the AGV was $3 \mathrm{~m} / \mathrm{s}$, however, to ensure safety of the experimental drives, the value of the reference velocity was maintained approximate by a human user. To reduce significant inaccuracies due to the approximate velocity values during the experimental drives, the AGV velocity was one of the real time input parameters of the used kinematic control strategy. 


\subsection{Path planning in unstructured environment}

The results of the theoretical and experimental application of the algorithm in the unstructured environment are respectively given in Fig. 2-4. During the first - the simple turn manoeuvre, the primary coarse path was made of three waypoints, i.e. two straight line segments and one sharp turn by 90 degrees (Fig. 2, part a). After the application of the proposed algorithm, the value of the optimised turning radius was 14 meters. From Fig. 2 (part a) it can be seen that the theoretically simulated movement of the AGV in the optimised path accurately matches the shape of the optimised path. Taking into consideration the results given in Fig. 2 (part b), it can be noted that the performed experimental drives also precisely match the shape of the optimised path. During the experimental drives of the simple turn manoeuvre, no major deviations were recorded. Thus, both the optimised path and the theoretical and experimental drives accurately match the straight-line segments, also the first and the final waypoints of the predefined primary coarse path.

During the second, the turnaround manoeuvre, the primary coarse path was made of four waypoints i.e. three straight line segments and two sharp turns, each by 90 degrees (Fig. 3, part a). From Fig. 3 it appears that only one optimisation problem was solved and respectively, the manoeuvre to turn by 180 degrees was performed. Despite this, the proposed algorithm operates in order that primarily, the first sharp turn $\left(w_{2}\right)$ was optimised, and after that, another sharp turn $\left(w_{3}\right)$ was optimised. Thus, based on the algorithm structure, optimisations of two sharp turns, each by 90 degrees, were performed. After the application of the proposed algorithm, the values of both optimised turning radiuses $\left(r_{1}\right.$ and $\left.r_{2}\right)$ were 11.5 meters. In this case, the beginning and the end of the optimised path also accurately match the first and the final waypoints of the predefined primary coarse path. However, from the results of the theoretically simulated movement of the AGV (Fig. 3, part a), it can also be seen that at the beginning of the movement in the last straight line segment (from $w_{3}$ to $w_{4}$ ) a deviation from the optimised path starts to emerge and at the final waypoint $\left(w_{4}\right)$ reaches 0.4 meters. The analysis of the simulation data showed that the deviation from the predefined path starts to emerge not because of the fault of the developed path planning algorithm, but due to how the used kinematic control strategy operates. In order to ensure that the AGV would precisely follow the optimised path, while taking into consideration the AGVs velocity and the angular velocity of the steering wheel turning, in the used kinematic control strategy, the beginning point of the steering wheel turning from the neutral position to the desired value is respectively earlier than the theoretical one. Thus, during the movement in the first optimised radius $r_{1}$, no deviations occur. Due to the reason that in this case the optimised turning radiuses $r_{1}$ and $r_{2}$ are equal and at the beginning of the movement in the second optimised turning radius $r_{2}$ the steering wheel is being held in the same position. Deviations from the optimised path also do not occur. However, during the end of the manoeuvre the turning of the steering wheel back to its neutral position is not started sooner than predicted theoretically, therefore the steering wheel is returned to its neutral position with inaccuracy and deviations from the optimised path in the last straight-line segment start to emerge. The same observations can be made based on the third - the more complex manoeuvre. The third manoeuvre was made of six waypoints i.e. five straight line segments and four sharp turns, which values respectively were: 100.1, 79.9, 69.2 and 169.9 degrees (Fig. 4, part a). After the application of the proposed algorithm, the values of the optimised turning radiuses were: $r_{1}-14.85 \mathrm{~m}, r_{2}-9.62 \mathrm{~m}, r_{3}-7.4 \mathrm{~m}, r_{4}-15.24 \mathrm{~m}$. As in the previous manoeuvre case (Fig. 3, part a), in this manoeuvre case (Fig. 4, part a) similarly, the beginning and the end of the optimised path accurately match the first and the final waypoints of the predefined primary coarse path. The results of the theoretically simulated movement of the AGV also show that the deviation from the optimised path starts to emerge with the same pattern, due to the used kinematic control strategy and not because of the developed path planning algorithm or path infeasibility.

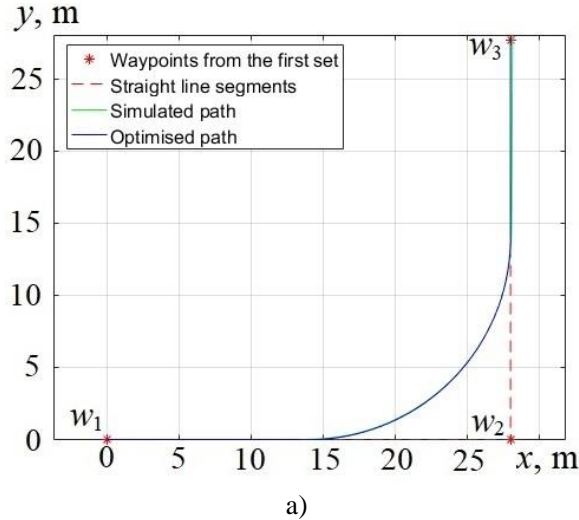

a)

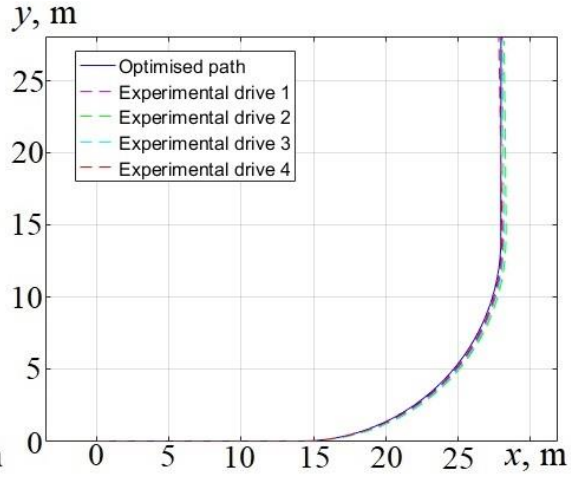

b)

Figure 2. Simple turn manoeuvre: $\mathrm{a}$ - theoretical results; $\mathrm{b}$ - experimental results 
From Fig. 3 (part b) and Fig. 4 (part b) it can be noticed that the deviations from the optimised path also occurred during the experimental drives of both turnaround and the more complex manoeuvres. While not taking into consideration the already described problem with the used kinematic control strategy, based on the given data of the experimental results (Fig. 3, part b and Fig. 4, part b), another remark can be pointed out. As can be seen from the mentioned figures, in every experimental drive during both manoeuvres, deviations were faintly different. Although, to reduce significant inaccuracies, the AGV velocity was one of the real time input parameters of the used kinematic control strategy, the control strategy was formulating steering control signals only based on the travelled distance. This means that every part of the manoeuvres was begun to perform after precisely evaluating the influence of the varying velocity on the travelled distance during the manoeuvre, however without evaluating the influence of the varying velocity on the accurate movement direction, i.e. the angle of the straight-line segment. Thus, it can be assumed that the different deviations in the experimental drives were formed by the varying velocity of the $\mathrm{AGV}$, because it is considered that, in the proposed hybrid global path planning algorithm, the reference velocity of the $\mathrm{AGV}$ is constant. Regarding this assumption, it can be noted that the deviations from the optimised path which occurred during the experimental drives are also the result of the kinematic control strategy.

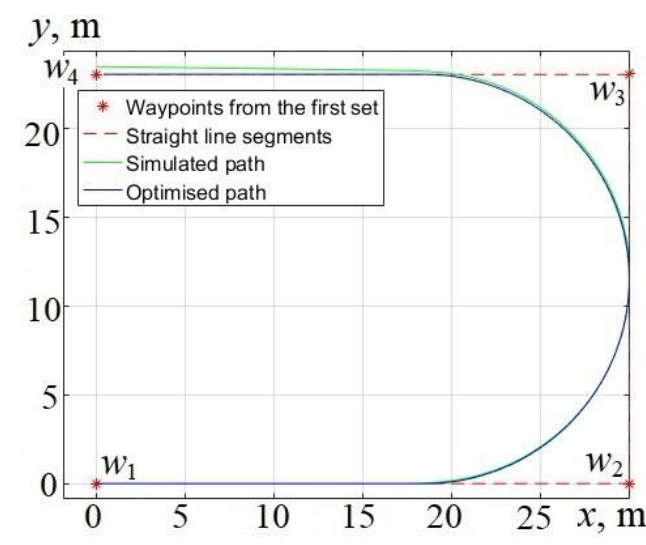

a)

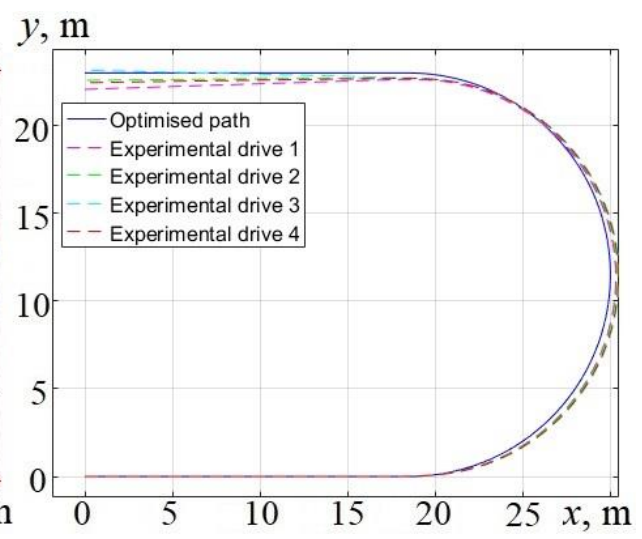

b)

Figure 3. Turnaround manoeuvre: $\mathrm{a}$ - theoretical results; $\mathrm{b}$ - experimental results

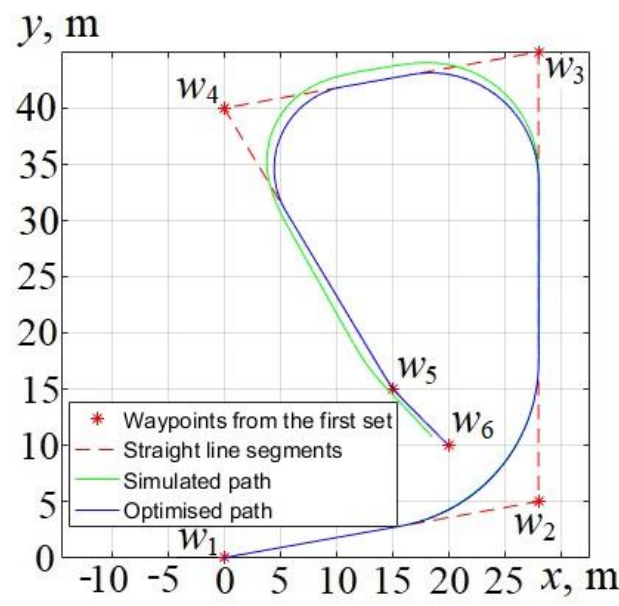

a)

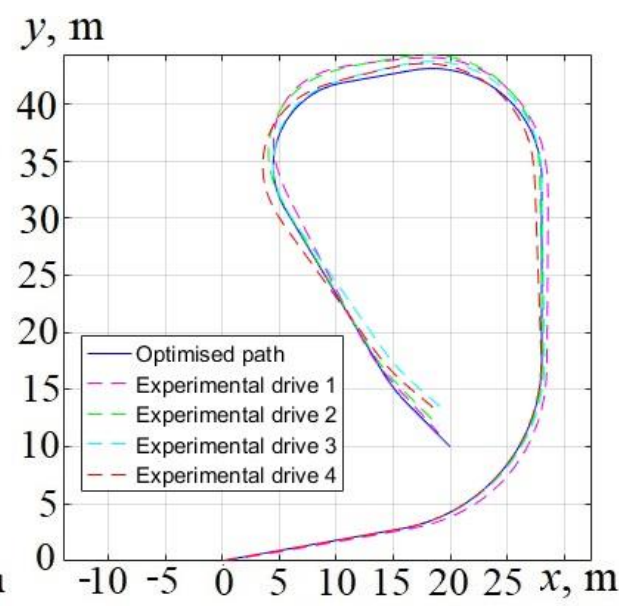

b)

Figure 4. Complex manoeuvre: $\mathrm{a}$ - theoretical results; $\mathrm{b}$ - experimental results

As can be seen from the provided results, the theoretical and experimental application of the algorithm in the unstructured environment singled out the drawbacks of the used kinematic control strategy and not the drawbacks of the proposed hybrid global path planning algorithm. It can also be seen that in all the cases of the application of the algorithm, the beginning, the end and the straight-line segments of the optimised path accurately match the corresponding waypoints and moving directions of 
the predefined primary coarse path. Furthermore, the results given in Fig. 2 (part a and b), Fig. 3 (part a) and Fig. 4 (part a), indicate that in particular parts of every manoeuvre during the theoretical simulations and the experimental drives, when the used kinematic control strategy did not cause deviations from the predefines path, the AGV was able to accurately follow the optimised path. Based on these observations it can be stated that the proposed hybrid algorithm performs properly while planning a path in an unstructured environment and ensures that the generated path would be smooth and dynamically feasible.

\subsection{Path planning in structured environment}

As in the previous section, the results of theoretical and experimental application of the algorithm in the structured environment are given in Fig. 5-8. As already noted, during this stage of the algorithm analysis, the algorithm was applied by selecting and recreating the manoeuvring in four different intersections, evaluating the geometry of the intersections. The intersections were selected based on the possibility to determine the geometrical parameters of the intersections as accurately as possible. The measured geometrical parameters of the intersections, the angles of the sharp turns, the optimised turning radius values and other parameters are given in Table. 1.

Table 1. Parameters of the selected intersections

\begin{tabular}{|c|c|c|c|c|}
\hline \multirow{2}{*}{ Parameter } & \multicolumn{4}{|c|}{ Value } \\
\hline & Intersection I & Intersection II & Intersection III & Intersection IV \\
\hline Direction & \multicolumn{2}{|c|}{ Turning to the left } & \multicolumn{2}{|c|}{ Turning to the right } \\
\hline Angle of sharp turn, & 111.8 & 99.64 & 81.38 & 95.79 \\
\hline$I_{i}, \mathrm{~m}$ & 11.5 & 25.3 & 17.1 & 21.5 \\
\hline$T_{i}, \mathrm{~m}$ & 5.62 & 10.94 & 9.94 & 10.65 \\
\hline$S D_{i}, \mathrm{~m}$ & \multicolumn{4}{|c|}{1.5} \\
\hline$r_{i}, \mathrm{~m}$ & 13.38 & 26.27 & 15.81 & 22.68 \\
\hline
\end{tabular}

Taking into account that in this case the aim of the application of the algorithm was to ensure that the performance of the newly formulated system of inequality constraints is proper, i.e. the road network limitations were not damaged, the deviations from the optimised path due to the used kinematic control strategy are not furtherly discussed. From the provided theoretical results it can be seen that during the path optimisation in every intersection, the beginning and the end of the optimised paths accurately match the first and the final waypoints of the primary coarse path. Respectively, the optimised angles and directions of the straight-line segments precisely match the predefined directions. It is important to remark that the optimised path did not damage any of the intersections geometrical limitations (Fig. 5-8, part a). Thus, it can be indicated that the optimisation process works properly and without any faults, while applying the proposed hybrid global path planning algorithm with the newly developed system of inequality constraints for structured environments.

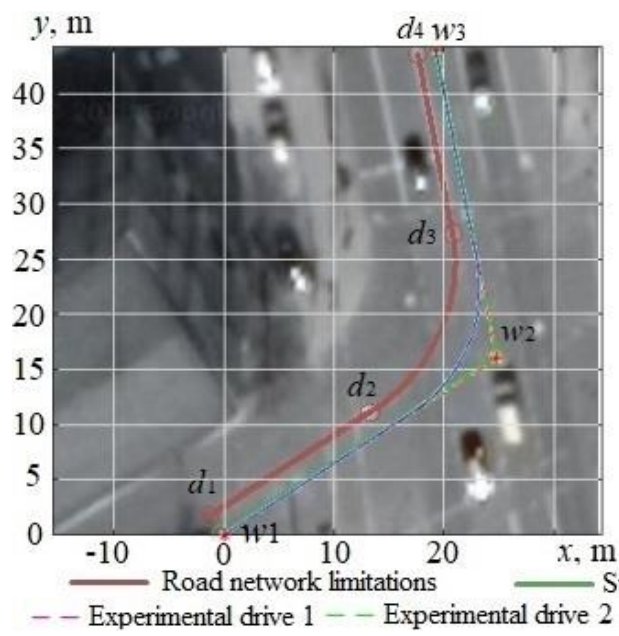

a)

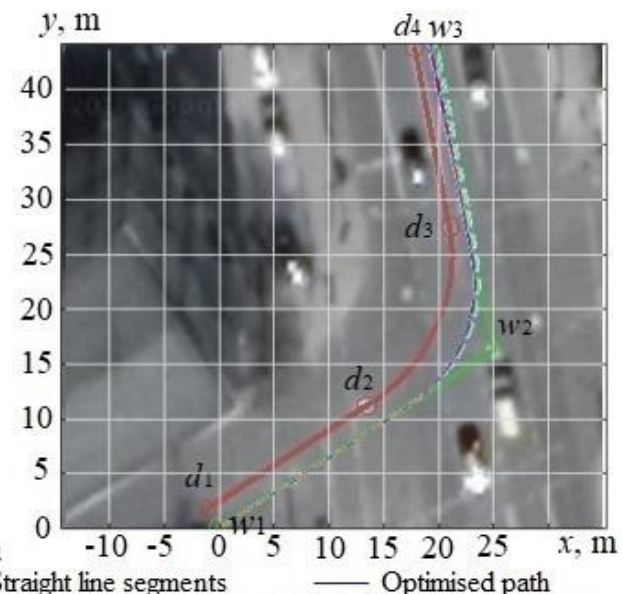

b)

Figure 5. Intersection I: a - optimisation results; b-experimental results 


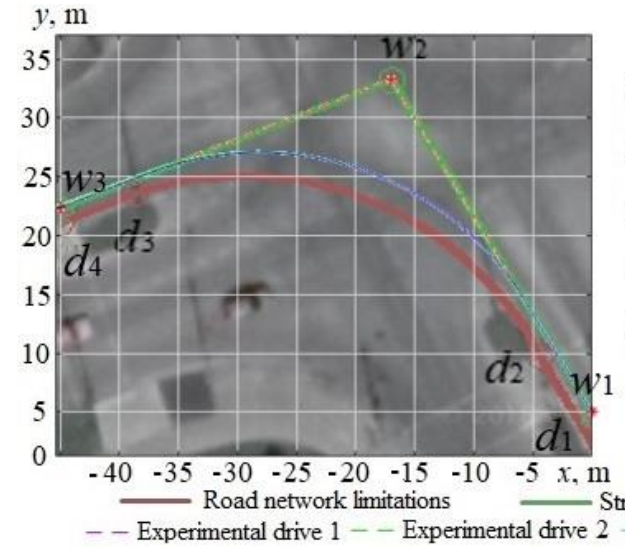

a)

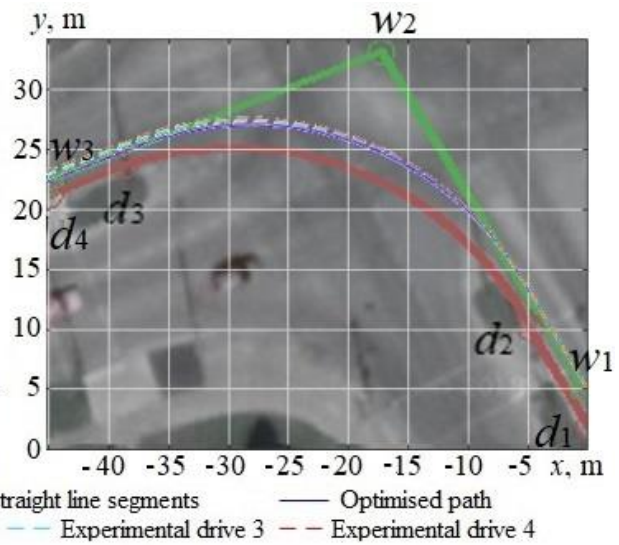

b)

Figure 6. Intersection II: $\mathrm{a}$-optimisation results; $\mathrm{b}$ - experimental results

Considering the results of the experimental drives (Fig. 5-8, part b), it can be noted that during the movement in all the intersections, there are no clearly visible deviations from the optimised paths. Furthermore, it can be pointed out that in all the experimental drives, the experimental movement curves match / overlap. From all of the optimised paths beginnings to the final waypoints, the autonomous ground vehicle was successfully following the optimised paths with different optimised turning radius values both during turning to the left and turning to the right, while evaluating different geometrical limitations, and did not collide with any of the cones, which imitated the road network limitations.

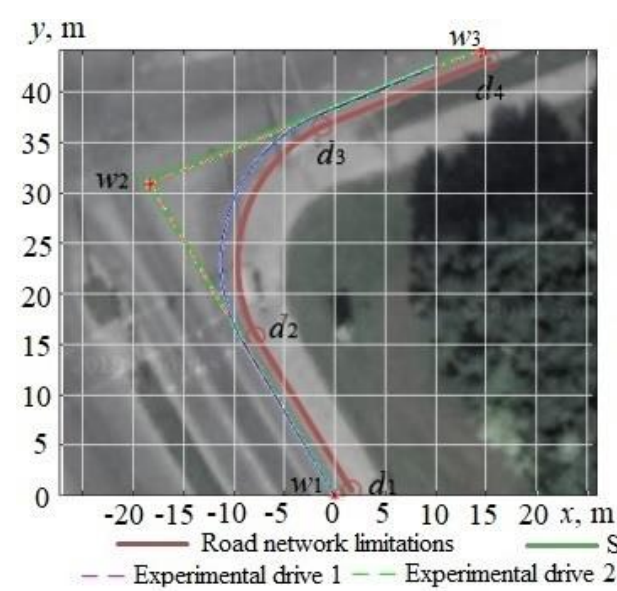

a)

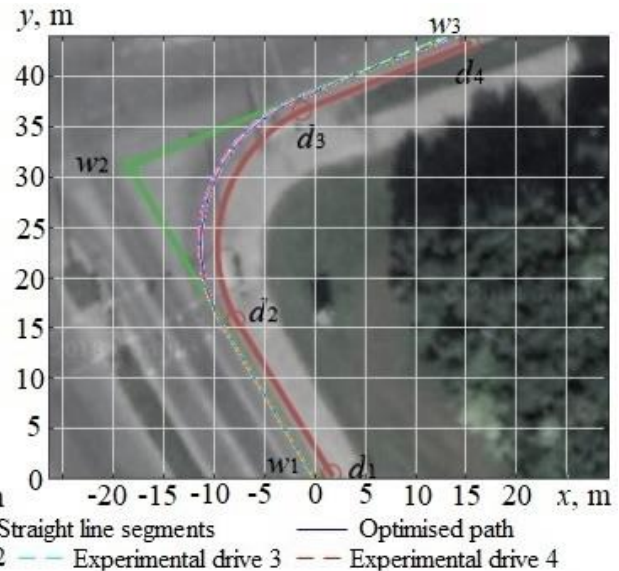

b)

Figure 7. Intersection III: a - optimisation results; b-experimental results

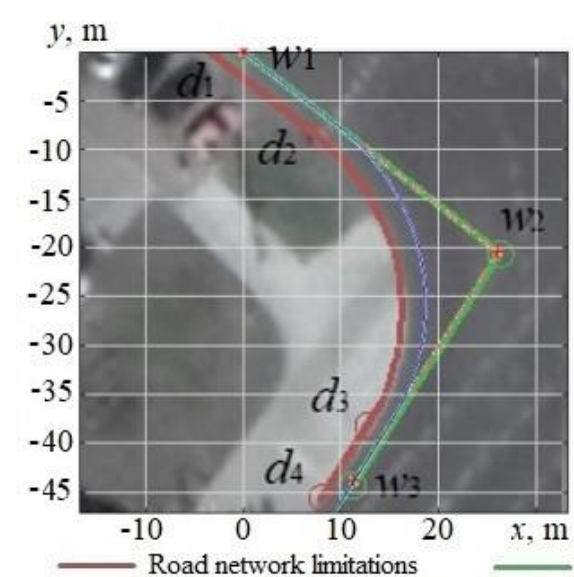

Road network limitations

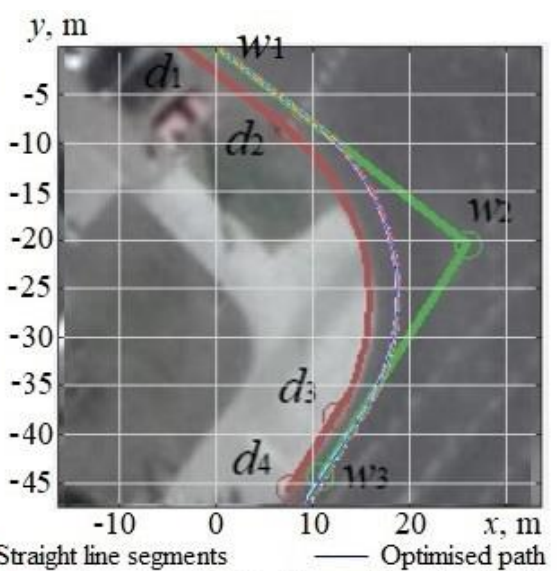

- Experimental drive 1 - Experimental drive $2-$ Experimental drive $3-$ - Experimental drive 4

a)

b)

Figure 8. Intersection IV: a - optimisation results; b - experimental results 
Such results of the algorithm application prove that the proposed hybrid global path planning algorithm also performs properly in structured environments, while taking into consideration different geometric parameters and limitations for the path optimisation. Thus, it can be stated that the described algorithm with the newly developed inequality constraints for a structured environment, can generate a smooth, dynamically feasible and most importantly a collision-free path, i.e. the algorithm ensures that the road network limitations would not be damaged.

\section{Conclusions}

In this work the development process, including the explanation of made assumptions, the detailed pattern of the algorithm application, also the theoretical and experimental based analysis of the hybrid global path planning algorithm are given. The described hybrid path planning algorithm is combined from the classical - Dubins path, and the heuristic - genetic algorithm approaches. Firstly, the primary coarse path is converted into the Dubins path and secondly, the Dubins path is optimised by applying the genetic algorithm. The main contribution of this work to the described algorithm is not only the analysis and the evaluation of the algorithm, but also the newly developed inequality constraints for the path planning in a structured environment, which take into consideration the geometric limitations of the environment and do not allow to ignore these limitations. The theoretical analysis of the algorithm showed that regardless of the complexity of the primary coarse path, the algorithm works properly and generates an optimal path for the AGV. It is important to note that the deviations from the optimised path observed during the experimental drives can be explained by the fault of the used kinematic control strategy and not by the possible drawbacks of the proposed hybrid global path planning algorithm. While taking into consideration the theoretical and the experimental analysis results and setting aside the deviations from the optimised path, which were influenced by the used kinematic control strategy, it can be stated that the described algorithm performs properly in both unstructured and structured environments. Thus, the main advantage of the described hybrid global path planning algorithm with the newly developed inequality constraints can be pointed out - the algorithm works properly and can be applied for a smooth and dynamically feasible path planning both in unstructured and in structured environments. However, the drawback of the described algorithm, which can be explained by the Dubins path approach, also must be pointed out - during the optimisation only the steady state cornering condition is considered. Due to this reason, future work by the authors, is to improve the proposed algorithm by combining it with local path planning algorithm, in order to adjust the algorithm for the path planning in structured environments with static and dynamic obstacles, while considering not only the steady state cornering condition

\section{References}

1. Araujo de Lemos, R., Garcia, O., Ferreira, J.V. (2015) Local and Global Path Generation for Autonomous Vehicles Using Splines. In: Workshop on Engineering Applications - International Congress on Engineering (WEA), Bogota, October 2015. Colombia: IEEE, pp. 1-6.

2. Ayawli, B.B.K., Chellali, R., Appiah, A.Y., Kyeremeh, F. (2018) An Overview of Nature-Inspired, Conventional, and Hybrid Methods of Autonomous Vehicle Path Planning. Journal of Advanced Transportation, 1-27. DOI: 10.1155/2018/8269698

3. Chaari, I., Koubaa, A., Benaceur, H., Ammar, A., Alajlan, M., Youssef, H. (2017) Design and performance analysis of global path planning techniques for autonomous mobile robots in grid environments. International Journal of Advanced Robotic Systems, 14(2), 1-15.

4. Elhoseny, M., Tharwat, A., Hassanien, A.E. (2018) Bezier Curve Based Path Planning in a Dynamic Field using Modified Genetic Algorithm. Journal of Computational Science, 25, 339-350.

5. Gonzales, D., Perez, J., Milanes, V., Nashashibi, F. (2015) A Review of Motion Planning Techniques for Automated Vehicles. IEEE Transactions on Intelligent Transportation Systems, 17(4), 11351145. DOI: 10.1109/TITS.2015.2498841

6. Kikutis, R., Stankūnas, J., Rudinskas, D., Masiulionis, D. (2017) Adaptation of Dubins Paths for UAV Ground Obstacle Avoidance When Using a Low Cost On-Board GNSS Sensor. Sensors, 17(10), 1-23.

7. Lamini, C., Benhlima, S., Elbekri, A. (2018) Genetic Algorithm Based Approach for Autonomous Mobile Robot Path Planning. Procedia Computer Science, 127, 180-189. DOI: 10.1016/j.procs.2018.01.113

8. Lee, J.W., Lee, D.H, Lee, J.J. (2011) Global Path Planning Using Improved Ant Colony Optimization Algorithm through Bilateral Cooperative Exploration. In: $5^{\text {th }}$ IEEE International 
Conference on Digital Ecosystems and Technologies, Daejeon, June 2011. Korea: IEEE, pp. 109113.

9. Mac, T.T., Copot, C., Tran, D.T., De Keyser, R. (2017) A hierarchical global path planning approach for mobile robots based on multi-objective particle swarm optimization. Applied Soft Computing, 59, 68-76. DOI: 10.1016/j.asoc.2017.05.012

10. Noreen, I., Khan, A., Habib, Z. (2016) Optimal Path Planning using RRT* based Approaches: A Survey and Future Directions. International Journal of Advanced Computer Science and Applications, 7(11), 97-107. DOI: 10.14569/IJACSA.2016.071114

11. Paniagua, A.H., Bandera, J.P., Ruiz-de-Quintanilla, M., Bandera, A. (2018) Quad-RRT: A real-time GPU-based global path planner in large-scale real environments. Expert Systems with Applications, 99, 141v154. DOI: 10.1016/j.eswa.2018.01.035

12. Samadi, M., Othman, M.F. (2013) Global Path Planning for Autonomous Mobile Robot using Genetic Algorithm. In: International Conference on Signal-Image Technology \& Internet-Based Systems, Kyoto, December 2013. Japan: IEEE, pp. 726-730.

13. Zhang, Y., Chen, H., Waslander, S.L., Gong, J., Xiong, G., Yang, T., Liu, K. (2018) Hybrid Trajectory Planning for Autonomous Driving in Highly Constrained Environments. IEEE Access, 6 , 32800-32819. DOI: 10.1109/ACCESS.2018.2845448.

14. Zhou, F., Gon, L. E. (2013) An Improved Path Planning for Mobile Robots. In: International Conference on Information Science and Cloud Computing Companion, Guangzhou, December 2013. China: IEEE, pp. 589-594. 